\title{
Correction to: Repurposing of antidiabetics as Serratia marcescens virulence inhibitors
}

\author{
Wael A.H. Hegazy ${ }^{1}$ (D) - Maan T. Khayat ${ }^{2} \cdot$ Tarek S. Ibrahim $^{2,3,4} \cdot$ Mahmoud Youns $^{5} \cdot$ Rasha Mosbah $^{6} \cdot$ \\ Wafaa E. Soliman ${ }^{7,8}$
}

Published online: 26 March 2021

(C) Sociedade Brasileira de Microbiologia 2021

\section{Correction to: Brazilian Journal of Microbiology https://doi.org/10.1007/s42770-021-00465-8}

In the original publication of the article, the last author's affiliation was incorrect. The correct affiliation appears as below:

1. Department of Biomedical Sciences, College of Clinical Pharmacy, King Faisal University, AL AHSA 31982, Kingdom of Saudi Arabia

2. Microbiology and Immunology Department, Faculty of Pharmacy, Delta University for Science and Technology, Gamasa 35712, Egypt

Publisher's note Springer Nature remains neutral with regard to jurisdictional claims in published maps and institutional affiliations.

The online version of the original article can be found at https://doi.org/ $10.1007 / \mathrm{s} 42770-021-00465-8$

Wael A.H. Hegazy

waelmhegazy@daad-alumni.de

1 Department of Microbiology and Immunology, Faculty of Pharmacy, Zagazig University, Zagazig, Egypt

2 Department of Pharmaceutical Chemistry, Faculty of Pharmacy, King Abdulaziz University, Jeddah 21589, Saudi Arabia

3 Center of Excellence for Drug Research \& Pharmaceutical Industries, King Abdulaziz University, Jeddah 21589, Saudi Arabia
Department of Pharmaceutical Organic Chemistry, Faculty of Pharmacy, Zagazig University, Zagazig 44519, Egypt

5 Department of Biochemistry and Molecular Biology, Faculty of Pharmacy, Helwan University, Cairo, Egypt

6 Infection control Unit, Zagazig University Hospitals, Zagazig University, Zagazig 44519, Egypt

7 Department of Biomedical Sciences, College of Clinical Pharmacy, King Faisal University, AL AHSA 31982, Kingdom of Saudi Arabia

8 Microbiology and Immunology Department, Faculty of Pharmacy, Delta University for Science and Technology, Gamasa 35712, Egypt 